\title{
Influence of Gender and Age on the Performance of Chest Compression by Anesthesiologists - Data from Departmental Training Using the RQI System
}

Kerpel Asaf ${ }^{1}$, Yahav Dana ${ }^{1}$, Cohen Yitzhak ${ }^{1}$, Ziv Amitai ${ }^{2}$ Berkenstadt Haim ${ }^{1}$

1. Department of Anesthesiology, Sheba Medical Center, Tel Hashomer, Israel

2. National Center for Medical Simulation, Tel Hashomer, Israel

Background and Goal of Study:

The quality of cardiopulmonary resuscitation (CPR) is a major determinant of the survival from cardiac arrest. The aim of the present study was to test the association between rescuers' gender, age and the accuracy of chest compressions among anesthesiologists.

\section{Materials and methods:}

60 anesthesiologists performed 3, 2 minutes cycles of chest compressions with 2 minutes rest between cycles. The RQI (Laerdal) realistic mobile simulation station was used for training and data collection. Data on - mean compressions depth (MCD), percent of deep enough compressions (DEC), percent of fully released compressions (FRC) and mean compressions rate (MCR) were collected.

Results and discussion:

\begin{tabular}{|c|c|c|c|c|c|c|c|c|c|c|c|}
\hline \multicolumn{4}{|c|}{$1^{\text {st }}$ session } & \multicolumn{4}{|c|}{$2^{\text {nd }}$ session } & \multicolumn{4}{|c|}{$3^{\text {rd }}$ session } \\
\hline $\begin{array}{l}\text { Mean } \\
\text { Depth } \\
\text { (cm) }\end{array}$ & $\begin{array}{c}\text { Deep } \\
\text { enough } \\
(\%)\end{array}$ & $\begin{array}{c}\text { Full } \\
\text { release } \\
(\%)\end{array}$ & $\begin{array}{c}\text { Mean } \\
\text { rate } \\
\text { (comp/m } \\
\text { in) }\end{array}$ & $\begin{array}{l}\text { Mean } \\
\text { Depth } \\
\text { (cm) }\end{array}$ & $\begin{array}{c}\text { Deep } \\
\text { enough (\%) }\end{array}$ & $\begin{array}{c}\text { Full } \\
\text { release } \\
(\%)\end{array}$ & $\begin{array}{l}\text { Mean rate } \\
\text { (comp/min) }\end{array}$ & $\begin{array}{c}\text { Mean } \\
\text { Depth } \\
(\mathrm{cm})\end{array}$ & $\begin{array}{c}\text { Deep } \\
\text { enough } \\
(\%)\end{array}$ & $\begin{array}{c}\text { Full } \\
\text { release } \\
(\%)\end{array}$ & $\begin{array}{l}\text { Mean rate } \\
\text { (comp/min }\end{array}$ \\
\hline 52.6 & 69.6 & 78.6 & 117.5 & 54.4 & 79.8 & 86.7 & 112.3 & 55.3 & 86.0 & 89.9 & 114.6 \\
\hline \pm 7.4 & \pm 36.9 & \pm 32.1 & \pm 19.2 & \pm 6.2 & \pm 30 & \pm 27 & \pm 20.4 & \pm 6.1 & \pm 25.7 & \pm 20 & \pm 17.2 \\
\hline
\end{tabular}

MCD and DEC increased from the 1 st to the 2 nd training session $(p<0.05)$.

MCD, DEC $(p<0.001)$ and FRC $(p<0.05)$ increased from the 1st to the 3rd training session.

There was no change in the MCR.

Gender differences:

Unlike male anesthesiologists $(n=42)$, female anesthesiologists $(n=18)$ did not improve their performance with training sessions. Men anesthesiologist performed better than female anesthesiologists did in $\operatorname{MCD}(53.9 \pm 6.8 \mathrm{~cm}$ vs. $49.5 \pm 4.4 \mathrm{~cm}, p<0.05 ; 56.2 \pm 4.4 \mathrm{~cm}$ vs $49.2 \pm 7.1 p<0.01$; $57.2 \pm 3.5 \mathrm{~cm} \mathrm{p}<0.001$, in the 1st, 2 nd and 3rd sessions respectively). and DEC $(75.1 \pm 35.5 \%$ vs $57.7 \pm 36.1 \%, 88.1 \pm 23$ vs $59.9 \pm 36.9,94.5 \pm 66.5$ vs $66.5 \pm 36.7 ; p<0.05$ in the 1 st, 2 nd and $3 r d$ sessions respectively).

Age differences:

While all 36 anesthesiologist younger than 50 years completed 3 training sessions, 3/24 older anesthesiologists were exhausted and quitted training following the 1st sessions. Both groups improved performance with training and there were no differences between age groups.

Conclusions: Data support previous published data on the influence of gender and age on the quality of CPR. Data also suggest that in future training at least three training sessions should be performed.

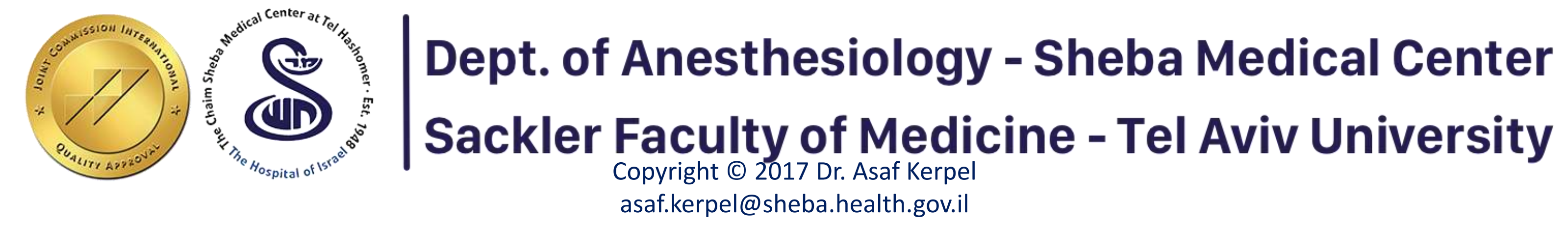

